\title{
X-Ray Luminescence of Crystallized Silver, Copper and Manganese-Doped Lithium Tetraborate
}

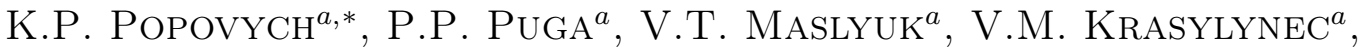 \\ V.M. HOLOVEY ${ }^{a}$ AND G.D. PUGA ${ }^{b}$ \\ ${ }^{a}$ Institute of Electron Physics, National Academy of Sciences of Ukraine \\ 21 Universitetska str., 88017 Uzhgorod, Ukraine \\ ${ }^{a}$ Uzhgorod National University, 46 Pidgirna str., 88017 Uzhgorod, Ukraine
}

\begin{abstract}
The X-ray luminescence spectra of doped lithium tetraborate have been analyzed on the basis of accounting the set of spectral transitions for different atomic charge states. It has been shown that the X-ray luminescence peculiarities are to a great extent due to the transitions between the energy levels of dopant atoms and ions, as well as to the levels, which form the lithium tetraborate matrix.
\end{abstract}

PACS numbers: 78.55.Qr, 71.55.Ak

\section{Introduction}

It is well known that introduction of a small quantity of dopant into the lithium tetraborate (LTB) enables its sensitivity to ionizing radiation influence to be increased substantially. The most promising from this point of view are the copper, silver and manganese dopants, for which the best photoluminescence characteristic indices were achieved [1-9] dependent on the criteria of the choice of the dopant type. In the most, such studies have been carried out for the monocrystalline lithium tetraborate-based phosphorus.

We suggest here a new approach to the analysis of the luminescence spectra of doped LTB in accordance with the peculiarities of the spectral composition of emission for different charge states of atoms. This method could be applied to explain the X-ray luminescence (XL) spectra and also the LTB matrix, the luminescence centers of which could be proper defects, excess or undercoordinated atoms of the structural network. Such approach is productive only for the broad-band materials, when the sufficient number of atomic levels of dopant-defect are located in the forbidden band, as well as given the assumption that the crystalline field affects insufficiently their energy values and does not result in the above levels splitting.

\section{Experimental results}

Note that the choice of a type of a dopant for the matrix based on the LTB materials affects considerably their luminescent characteristics and prospects of their use as the tissue-equivalent dosimetric materials. These properties are due to the energy spectrum of impurity states

\footnotetext{
* corresponding author; e-mail: kostyn@gmail.com
}

in the conduction band, which, in the case of the broad-band dielectrics, has its own specific features.

Indeed, in the case of LTB one should take into account that a large band gap $\left(E_{\mathrm{g}} \approx 9 \mathrm{eV}\right)$ realizes a specific "transparency window" for the external radiation, which, in the case of the presence of the discrete energy levels of the dopant atom or proper defects, is decisive for stimulated optical transitions. The question is how the structure of atomic levels of the dopant in the crystalline field of the substance matrix is preserved, how are they split and do they lose the signs of isolated atoms or ions?

It is well known that the valence electrons located in the unfilled electron shells take part in the energy band formation in semiconductors and dielectrics. For instance, it has been found in the case of LTB [10] that the electron states near the valence band vertices are formed mainly by the $2 p$-states of oxygen, while the deeper states correspond to the $2 s$ orbitals of oxygen. Contribution of atomic orbitals of other atoms $\left(\mathrm{Li}, \mathrm{B} 1\right.$ in $\mathrm{BO}_{3}$ and $\mathrm{B} 2$ in $\mathrm{BO}_{4}$ ) is 10 times less, that of the $2 p$-states of boron atoms dominates at the conduction band bottom, while $\mathrm{Li}$ and $\mathrm{B}$ atom orbitals take almost no part in the low-energy "band-band" transitions, since their contribution into transitions near the conduction band bottom is not essential.

Other (deeper) boron and oxygen atomic levels in the LTB matrix do not take part in the band structure formation, however, being filled with electrons, they also do not take part in the optical transitions. This situation is changed when the proper defects are produced or when the dopant atoms are incorporated into the matrix. In this case, the dopant atom levels occurring in the "transparency window" energy range may play a decisive role in the stimulated optical transitions in LTB. Moreover, they may determine the doped LTB luminescent spectra, i.e., the latter may have the signs of atomic or ionic emis- 
sion spectra of relevant dopant atoms. This is confirmed by the comparison of the luminescent spectra of doped $\mathrm{Li}_{2} \mathrm{~B}_{4} \mathrm{O}_{7}\langle\mathrm{Ag}, \mathrm{Cu}, \mathrm{Mn}\rangle$ with the energy positions of spectral lines of emission of the centers of atomic $\mathrm{Li}, \mathrm{B}, \mathrm{Ag}$, $\mathrm{Cu}$ and $\mathrm{Mn}$ in different charge states produced under the influence of high-energy exciting radiation.

The XL-spectra were excited under irradiation of the quanta up to the $40 \mathrm{keV}$ energy and $20 \mathrm{~mA}$ current by means of an X-ray tube with molybdenum anti-cathode.
The XL spectra studies were carried out using an automated setup according to the method described in [11]. The LTB samples crystallized from the glassy state at the $773 \mathrm{~K}$ temperature during $5 \mathrm{~h}$ were used in our studies including those doped by copper, silver and manganese. An activator quantity was chosen from the condition of the most intense XL spectra (i.e., $\mathrm{Cu}-0.056 \mathrm{~mol} \%$, $\mathrm{Ag}$ $-0.083 \mathrm{~mol} . \%$ and $\mathrm{Mn}-0.16 \mathrm{~mol} . \%)$.

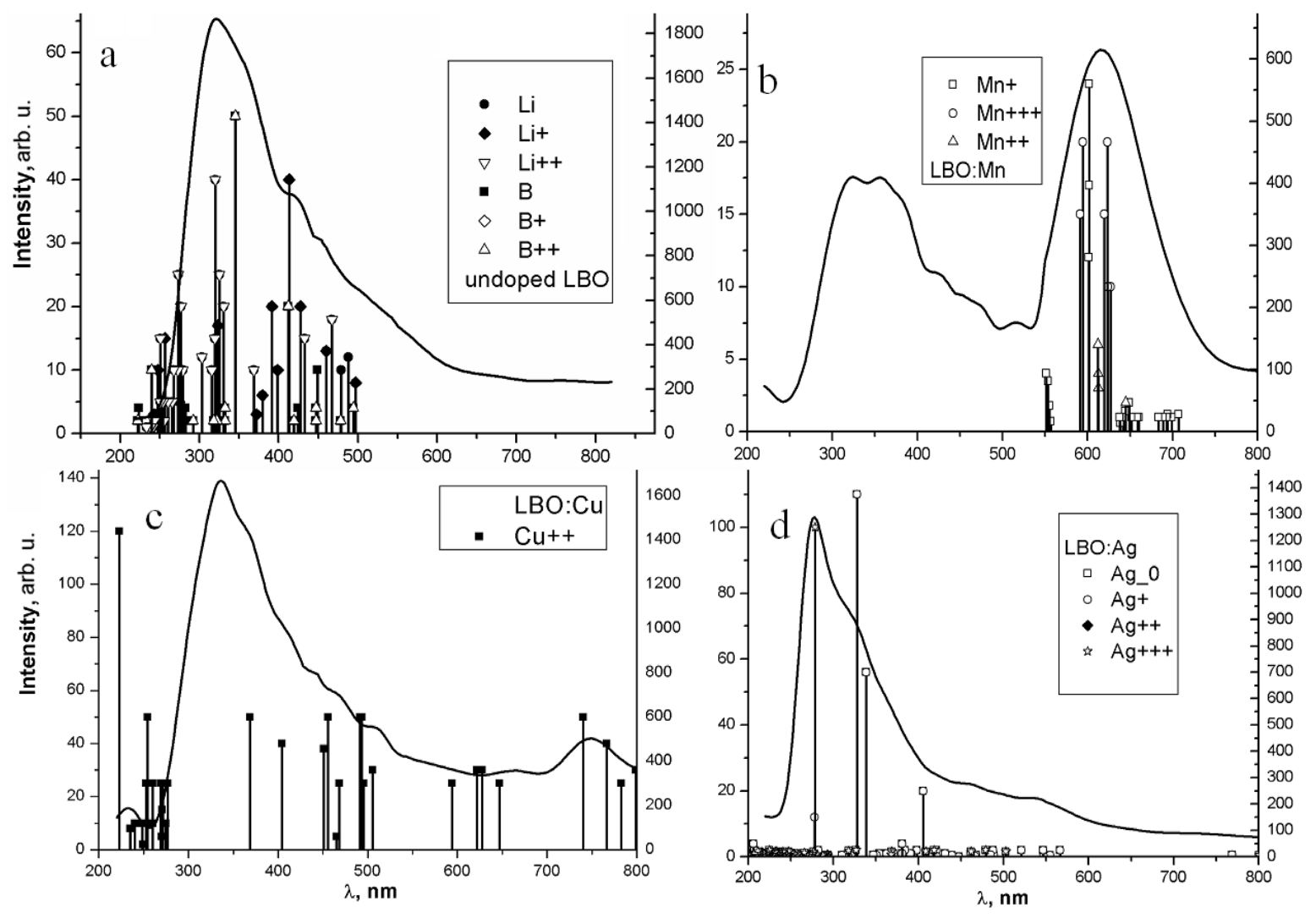

Fig. 1. X-ray luminescence spectra of LTB: (a) - undoped; (b) - Mn-doped; (c) - Cu-doped; (d) - Ag-doped. The plots show the position of emission lines for $\mathrm{Li}, \mathrm{B}, \mathrm{Mn}, \mathrm{Cu}$ and $\mathrm{Ag}$ atoms and ions. The left scale stands for the intensities of atomic lines. The right scale stands for the X-ray luminescence intensities.

The results of the studies are shown in Figs. 1a-d. As follows from Fig. 1a, the XL spectrum of the crystallized glassy $\mathrm{Li}_{2} \mathrm{~B}_{4} \mathrm{O}_{7}$ is characterized by a single broad band with a maximum at $322 \mathrm{~nm}(3.86 \mathrm{eV})$ and distinct features with the energies of $355 \mathrm{~nm}(2.96 \mathrm{eV}), 426 \mathrm{~nm}$ $(2.72 \mathrm{eV})$ and $540 \mathrm{~nm}(2.34 \mathrm{eV})$. Two main bands were observed in the XL spectrum of the crystallized LTB:Mn (Fig. 1b): a narrow one at $615 \mathrm{~nm}(2.02 \mathrm{eV})$ and a complex wide band with the maxima at: $375 \mathrm{~nm}(3.25 \mathrm{eV})$, $354 \mathrm{~nm}(3.46 \mathrm{eV})$ and $324 \mathrm{~nm}(3.83 \mathrm{eV})$. In the XL spectrum of the crystallized LTB:Cu (Fig. 1c), three distinct maxima were found at: $745 \mathrm{~nm}(1.66 \mathrm{eV}), 336 \mathrm{~nm}$ $(3.69 \mathrm{eV})$ and $232 \mathrm{~nm}(5.30 \mathrm{eV})$. It should be noted that the intense maximum in the $250-590 \mathrm{~nm}(2.10-4.8 \mathrm{eV})$ region has a complex nature.
The XL spectrum of LTB:Ag (Fig. 1d) is characterized by a broad band with a pronounced maximum at $278 \mathrm{~nm}$ $(4.46 \mathrm{eV})$ and a number of less intense features at $324 \mathrm{~nm}$ $(3.90 \mathrm{eV}), 462 \mathrm{~nm}(2.66 \mathrm{eV})$ and $542 \mathrm{~nm}(2.28 \mathrm{eV})$. Note that introduction of $\mathrm{Ag}$ modifier results in a shift of the main maximum to the UV region with respect to undoped crystallized lithium tetraborate, whereas doping with $\mathrm{Mn}$ and $\mathrm{Cu}$ results in its shift to the IR region of the spectrum.

Let us analyze the XL spectra of doped LTB on the basis of peculiarities of the dopant atoms spectra for different charge states.

From the analysis of the energy dependences under study (Fig. 1) it follows that a broad asymmetric emission band in the $250-500 \mathrm{~nm}(2.48-4.96 \mathrm{eV})$ region is 
typical for all of the above dependences. A considerable width and a specific shape of the band indicate its complex structure, while its position is related to the emission processes due to the crystallized LTB lattice with no participation of the dopant atoms (ions). The correctness of this assumption is justified by the identity of the shape and intensity of this band as compared to the emission spectrum of the LTB single crystal obtained in the similar conditions of excitation and existence of emission in this energy interval at doping by $\mathrm{Ag}$ or $\mathrm{Cu}$ [5]. Correlation between the positions of maxima of the band structure and the spectral position of atomic, singly and doubly-charged lithium lines allows one to suggest that this band in the undoped LTB polycrystal is due to the complexes comprising $\mathrm{Li}$ ions and atoms with emission energies corresponding to: $2 s 3 d-1 s 6 f, 1 s 3 s-1 s 4 p, 1 s^{2} 2 p$ $1 s^{2} 5 d$ and $1 s 3 d-1 s 4 f$ transitions. A certain contribution to this band is due to the boron atoms and ions, since some spectral emission lines coincide with the emission frequencies for the $1 s^{2} 2 s 2 p-1 s^{2} 2 p^{2}, 1 s^{2} 2 s 3 d-1 s^{2} 2 s 4 f$ and $1 s^{2} 2 s 3 d-1 s^{2} 2 s 4 f$ transitions [12].

In the case of the crystallized LTB:Mn, the XL spectrum demonstrates an intense maximum at $615 \mathrm{~nm}$ $(2.02 \mathrm{eV})$. A good agreement of the shape and energy position of this emission band with the radioluminescence emission spectrum [13] of polycrystalline LTB:Mn and photoluminescence spectra in the glassy and crystalline LTB:Mn [1] allows one to assert that the luminescence nature in this energy region is due to the radiative transitions of singly, doubly and triply-charged $\mathrm{Mn}^{+}$ions, the principal of them being as follows: $1 s 3 d-1 s 6 f, 3 d^{5} 4 s 4 p$ $3 d^{5} 4 s 5 s, 3 d^{5} 4 s 4 p-3 d^{5} 4 s 5 s$.

Similarly to the energy dependences described above, the LTB: $\mathrm{Cu}$ emission spectrum is characterized by an asymmetric broad maximum in the 250-550 nm $(2.25-4.96 \mathrm{eV})$ region, the luminescence of which is due to the emission of the centers that comprise the atoms of the basic matrix with no participation of the dopant atoms and ions. These are the radiative transitions $2^{2} S-$ $2^{2} \mathrm{P}$ due to the luminescence of singly-charged $\mathrm{Li}^{+}$and $\mathrm{B}^{+}$ions. Other features found in the XL spectrum for LTB: $\mathrm{Cu}$ in the $660-775 \mathrm{~nm}$ region $(1.6-1.88 \mathrm{eV})$ agree well by their energy positions with the emission frequencies of atoms on the doubly-charged copper ions due to the $3 d^{9} 4 s \rightarrow 3 d^{10}$ transitions [5].

The XL-spectrum of the crystallized LTB:Ag is very similar by the shape and intensity to the spectrum of stoichiometrically crystallized $\mathrm{Li}_{2} \mathrm{~B}_{4} \mathrm{O}_{7}$ and has a form of a broad band lying within the $250-600 \mathrm{~nm}(2.06-4.96 \mathrm{eV})$ interval. Large width and clear asymmetric character of this band indicate its complex structure. A considerable difference from the crystallized undoped LTB is a sharp short-wavelength limit of the band near $260 \mathrm{~nm}(4.77 \mathrm{eV})$. Such character of the energy dependence could be due to the imposition onto the XL spectrum of the emission of the crystallized $\mathrm{Li}_{2} \mathrm{~B}_{4} \mathrm{O}_{7}$ matrix with the luminescence maximum at $322 \mathrm{~nm}(3.85 \mathrm{eV})$ related to the emission of the dopant silver atoms or ions with the maximum at $278 \mathrm{~nm}(4.46 \mathrm{eV})$. This is also evidenced by the fact that the photoluminescence maximum of the monocrystalline LTB:Ag at 260-270 nm (4.77-4.59 eV) [4-6] and the glassy LTB:Ag at $300 \mathrm{~nm}(4.13 \mathrm{eV})$ [5] agrees well with the maximum of the polycrystalline LTB:Ag studied by us, while the nature of the luminescence in this region is due to the emission of $\mathrm{Ag}^{+}$ions at the $4 d^{9} 5 S \rightarrow 4 d^{10}$ transitions.

\section{Conclusions}

In accordance with the analysis of the energy dependences of XL for the crystallized $\mathrm{Li}_{2} \mathrm{~B}_{4} \mathrm{O}_{7}$-based materials doped by copper, manganese and silver, one may conclude that the X-ray luminescence in these materials is mainly due to the transitions between the energy levels of the dopant atoms and ions lying in the "transparency window" energy region 250-500 $\mathrm{nm}(2.48-4.96 \mathrm{eV})$ and the levels formed due to the ions and atoms, which form the LTB matrix.

\section{References}

[1] M. Ignatovych, V. Holovey, T. Vidoczy, P. Baranyai, A. Kelemen, Radiation Phys. Chem. 76, 1527 (2007).

[2] A. Kelemen, M. Ignatovych, V. Holovey, T. Vidoczy, P. Baranyai, Radiation Phys. Chem. 76, 1531 (2007).

[3] M. Ignatovych, V. Holovey, A. Watterich, T. Vidoczy, P. Baranyai, A. Kelemen, O. Chuiko, Radiation Measurements 38, 567 (2004).

[4] M. Ignatovych, V. Holovey, A. Watterich, T. Vidoczy, P. Baranyai, A. Kelemen, V. Ogenko, O. Chuiko, Radiation Phys. Chem. 67, 587 (2003).

[5] M. Ignatovych, V. Holovey, T. Vidoczy, P. Baranyai, A. Kelemen, V. Laguta, O. Chuiko, Funct. Mater. 12, 313 (2005).

[6] D. Shahare, B. Deshmukh, S. Moharil, S. Dhopte, P. Muthal, V. Kondawar, Phys. Status Solidi A 141, 329 (1994).

[7] V. Holovey, V. Sidey, V. Lyamayev, P. Puga, J. Lumin. 126, 408 (2007).

[8] V. Holovey, V. Lyamayev, M. Birov, P. Puga, A. Solomon, Funct. Mater. 12, 2 (2005).

[9] N. Senguttuvan, M. Ishii, M. Shimoyama, M. Kobayashi, N. Tsutsui, M. Nikl, M. Dusek, H.M. Shimizu, T. Oku, T. Adachi, K. Sakai, J. Suzuki, Nuclear Instruments and Methods in Physics Research A 486, 264 (2002).

[10] V.V. Maslyuk, M.M. Islam, T. Bredow, Phys. Rev. B 72, 125101 (2005).

[11] B. Hunda, Uzhgorod University Scientific Herald. Series Physics 5, 198 (1999).

[12] S.E. Frish, Optical Spectra of Atoms, Izv. Fiz. Mat., Moskva-Leningrad 1963, (in Russian).

[13] G. Enfurt, M. Krbetschek, J. Radiat. Prof. Dosim. 100, 403 (2002). 